\title{
Wave system fitting: A new method for force measurements in shock tunnels with long test duration
}

\author{
Changtong Luo*, Yunpeng Wang, Chun Wang, Zonglin Jiang \\ State Key Laboratory of High Temperature Gas Dynamics, Institute of Mechanics, Chinese Academy of Sciences, Beijing 100190, China
}

\section{A R T I C L E I N F O}

\section{Article history:}

Received 10 October 2014

Received in revised form

24 January 2015

Accepted 12 February 2015

Available online 3 April 2015

\section{Keywords:}

Aerodynamic force measurements

Strain gauge balance

Inertia compensation

Signal decomposition

Accelerometer-free

Shock tunnels

\begin{abstract}
A B S T R A C T
Force measurements in shock tunnels are difficult due to the existence of vibrations excited by a sudden aerodynamic loading. Accelerometer inertia compensation could reduce its negative effect to some extent, but has inherent problems. A new signal decomposition method, wave system fitting (WSF), is proposed to remove vibration waves of low frequency. The WSF is accelerometer-free. It decomposes the balance signal and can separate vibration waves without the influence on the DC component, and it does work no matter the cycle of the sample signal is complete or not. As a standard signal postprocessing tool in JF-12, the application results show that it works reliably with high accuracy, and it can also explain puzzling signals encountered in JF-12. WSF method is especially useful and irreplaceable whenever only a few cycles of a periodic signal could be obtained, as is usual for shock tunnels.
\end{abstract}

(c) 2015 Elsevier Ltd. All rights reserved.

\section{Introduction}

A new aircraft needs thousands of wind tunnel tests before it can really fly in the sky. For hypersonic vehicles, shock tunnels are the most promising ground-based-test facilities because they can provide a large range of stagnation enthalpy and flow velocities, and even duplicate hypersonic flight conditions. However, shock tunnels have a very short test duration (effective run-time), usually in milliseconds. This makes it very difficult to measure the aerodynamic forces. Classical strain gauge balance is used in our tests, though some other kind of balances such as accelerometer balance and stress-wave force balance system [12] might be feasible. In the beginning of the wind tunnel test, a sudden aerodynamic loading will excite the vibration of the model-balance-sting system, which can distort the balance signal sensed by strain gauges in force measurements. Special techniques must be applied to reduce the negative effect of the vibration to get the right result.

A practical and widely used solution is acceleration compensation $[1,3,8,9,11,13,14]$. That is, to embed some accelerometers into the balance in the hope that the vibration signal can be removed from the balance signal produced. But this method has inherent problems. First, the signal collected by the accelerometers depends on the global displacement of model-balance-sting system, but the strain gauges can only sense the local strains within the balance. In general, the global displacement and local strains might be inconsistent. In addition, the phase offset and partial compensation issues could also decrease the accuracy of force measurements.

\footnotetext{
* Corresponding author.

E-mail addresses: luo@imech.ac.cn (C. Luo), wangyp@imech.ac.cn (Y. Wang), wangchun@imech.ac.cn (C. Wang), zljiang@imech.ac.cn (Z. Jiang).
} 
In 2012, a super large shock tunnel, referred to as the Long-test-duration Hypervelocity Detonation-driven Shock Tunnel (JF-12) was developed [2]. JF-12 is capable of reproducing the pure air flow of flight conditions at Mach numbers from 5 to 9 and altitude of $25-50 \mathrm{~km}$ with at least $100 \mathrm{~ms}$ test duration. The long test duration brings much convenience for force measurements. The balance signal in JF-12 shows a periodic feature during the steady test phase. This enables us to develop a new method, wave system fitting (WSF), to analyze the aerodynamic force signals of strain gauge balance in shock tunnels with long test duration. The proposed method does not rely on any accelerometer, and derives aerodynamic forces directly from balance signals. The new method is accelerometer-free, so it does not have the problems caused by accelerometers to conventional strain gauge balances mentioned above.

\section{Wave system fitting}

\subsection{Dynamics of structures}

The test model used in this paper is a sharp cone of $10^{\circ}$ half-angle and $1.5-\mathrm{m}$ length, which is the largest sharp cone model so far in the world for force measurements in shock tunnels. We choose the sharp cone to test our force measurement method because it is a kind of standard test model which has been tested by several other hypersonic wind tunnels, and it has also an approximation solution with Newton's theory under laminar flow conditions. Thus, the results could be comparable.

The cone is connected with an inner strain gauge balance and a stiffness enhanced sting (Fig. 1). The connection detail of the three parts is shown in the lower left quarter of Fig. 1.

Structural analysis can help determine the vibration shapes and frequencies of the model-balance-sting system, and thus separate the signals of aerodynamic force and natural vibrations. For a simple structure such as a cantilever beam, it is not difficult to carry out its dynamic analysis. However, the structure of model-balance-sting systems used in wind tunnels for force measurements is usually quite complex, as shown in Fig. 1. Analytic solutions are not available any more. We have tried three different methods in this work to study the natural frequencies and mode shapes of vibration: finite element analysis (FEA), fast Fourier transform (fft) of knocking test signals and that of wind-tunnel test signals.

The balance signal of knocking and/or wind-tunnel tests involves many vibrations of high frequency. Usually, the amplitude of these high frequency vibrations is not so large. The total effect of a large number of these small vibrations behaves like white noise. The average of their displacement approaches zero, which means high frequency vibrations will not have much influence on the measurement accuracy. On the contrary, the balance signal also involves a few low frequency vibrations with large amplitude, which could affect the accuracy of measurement results if not properly handled. Therefore, we will focus only on low frequency vibrations in this paper. For the normal force of the sharp cone model, finite element analysis shows that the natural vibration frequencies of the model-balance-sting system are $24.8 \mathrm{~Hz}, 44.1 \mathrm{~Hz}, \ldots$, $147.9 \mathrm{~Hz}, \ldots, 241.6 \mathrm{~Hz}, 250.6 \mathrm{~Hz}, \ldots$, etc. While the fft of knocking test signals gives $48.6 \mathrm{~Hz}, 146.0 \mathrm{~Hz}, 243.3 \mathrm{~Hz}, \ldots$, etc., and the fft of wind-tunnel test signals gives $49.9 \mathrm{~Hz}, 239.5 \mathrm{~Hz}$ for shot \#20130422, and $49.9 \mathrm{~Hz}, 249.5 \mathrm{~Hz}$ for shot \#20130427 (see Fig. 2).

By knocking test with a plastic hammer, we find that only a few of frequency of vibrations could be excited in a single knock. For example, the vibrations of frequency $146.0 \mathrm{~Hz}$ and/or $243.3 \mathrm{~Hz}$ might not be excited in some knocks. But all frequencies excited by knocking could be found in FEA frequencies approximately. A wind-tunnel test behaves like a single knocking. So only a few of knocking-test frequencies would be excited in one shot, as shown in Fig. 2 . In summary, the inclusion relation of the three frequency set satisfies $\mathbb{F}_{\text {shot }} \subset \mathbb{F}_{\text {knock }} \subset \mathbb{F}_{\text {FEA }}$.

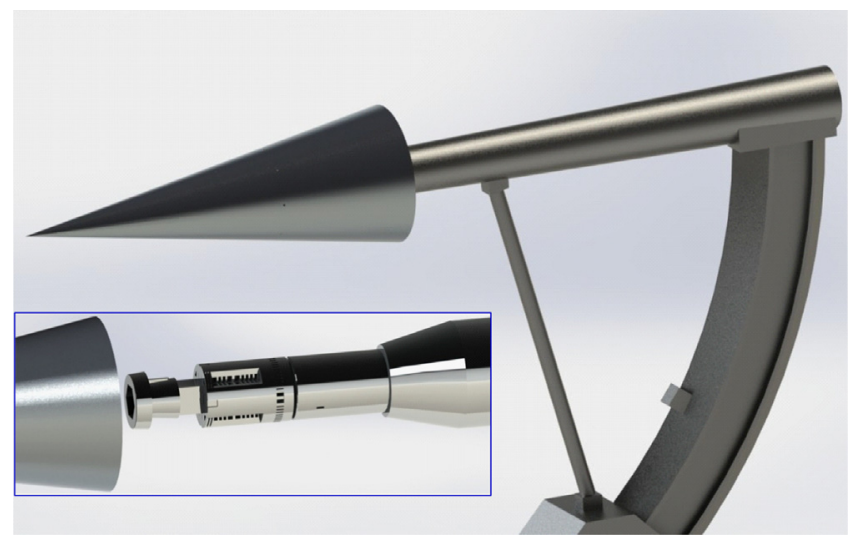

Fig. 1. The model-balance-sting system and its connection detail. 


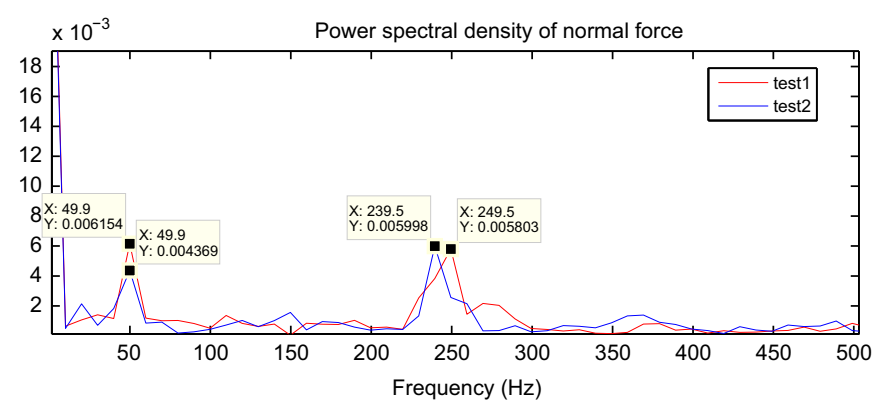

Fig. 2. Balance signals of normal force in frequency domain.

\subsection{Separation of vibration signals}

In JF-12, the vibration is caused by the starting up of the wind tunnel and flow establishing process. Usually it takes $20-30 \mathrm{~ms}$. This process is very complex. But in the next $100-120 \mathrm{~ms}$, the flow stream will be quite steady. The nominal 100 ms test duration refers to this $100 \sim 120 \mathrm{~ms}$ steady phase. At the beginning of this $100 \mathrm{~ms}$, the natural vibrations of the model-balance-sting system have been excited already, and the inertia force will last a long time, much more than the $100 \mathrm{~ms}$.

Based on these facts and the above structural analysis of the model-balance-sting system, we can conclude that the balance signal in the steady test phase mainly consists of two kinds: the direct current (DC) component of the aerodynamic force/moment with zero-frequency and the periodic components of inertia force with different frequencies.

Again, take the normal force component as an example, a typical balance signal is shown in Fig. 3(a). It shows that the wind-tunnel started up at about $110 \mathrm{~ms}$, and the test flow reached its steady state after about $20 \mathrm{~ms}$. So the next $100 \mathrm{~ms}$ signal (framed by a red rectangle in Fig. 3) is taken to calculate the desired aerodynamic force. The power spectral density of this part of signal (see Fig. 3(b)) shows that there exist at least two low frequency vibrations at $49.9 \mathrm{~Hz}$ and $249.5 \mathrm{~Hz}$ in the signal. The starting point is not zero. This means that there is also a zero-frequency component, known as direct current (DC) component, and that is exactly what we want to get, the signal of aerodynamic force.

Note that high frequency vibrations do not have much influence on the measurement accuracy, and it is enough to separate and remove some low frequency vibrations to get the right result with an acceptable accuracy.

The time-displacement of the balance signal could be modeled as follows:

$$
x(t)=f+\sum_{i=1}^{m} A_{i} \sin \left(2 \pi \omega_{i} t+\varphi_{i}\right)+\varepsilon
$$

where the first term $f$ is a constant, corresponding to the DC component of the signal (or the aerodynamic force), the second part represents the low frequency signals, and the last term $\varepsilon$ contains high frequency signals and random noise. The parameter $m$ in Formula (1) is a choice for the number of low frequency waves to be removed, and it should be determined with the experimental data for each test.

The wave system fitting involves the two steps as follows.

(a) m-determination: The second part involves $m$ sine waves with different amplitudes, frequencies and initial phases, which are referred to as wave system in this paper. The parameter $m$ could be determined by the power spectral density (or fft) of the balance signal and the required measurement accuracy. Keep in mind that low frequency waves affect the accuracy more than high ones. According to our experience, taking $m=1-4$, and usually $m=2$, is a good choice to get an acceptable result.

(b) Coefficient optimization: Once the parameter $m$ is determined, the wave system fitting to the balance signal could determine the coefficients in Formula (1). If all the coefficients including $f, A_{i}, \omega_{i}, \varphi_{i}$ and $\varepsilon$ are properly determined, low frequency signals could be removed, and the desired force signal could be approximated by $f+\varepsilon$.

\subsection{Optimization considerations}

The wave system fitting is a critical step in the decomposition of the balance signal to remove vibration signals. It could be considered as a special nonlinear fitting process to determine the coefficients in Formula (1). Note that these coefficients must be globally optimal. Otherwise, the result could be misleading. A fast and reliable global optimization (GO) algorithm is required for the decomposition of balance signal. The low dimensional simplex evolution (LDSE) [5-7] is chosen for this task 

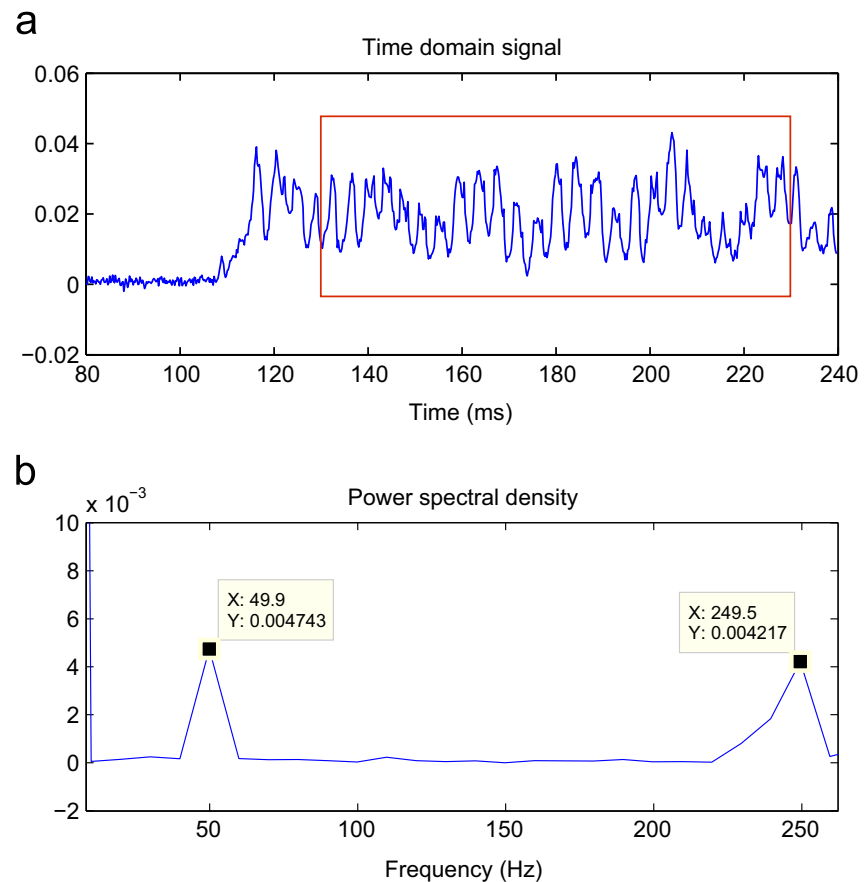

Fig. 3. A typical balance signal and its power spectral density. (a) Time domain signal of normal force, time interval: 80 - $240 \mathrm{~ms}$ (b) Power spectral density, time interval: $130-230 \mathrm{~ms}$.

in this work, because it is an efficient GO algorithm of our own, and we know every detail of its control parameters to ensure its global convergence.

\section{Inversion capability test of the new method}

The wave system fitting, involving $m$-determination and coefficient optimization, reconstructs the wave system using sample points. In this sense, it is a kind of inverse problem. It is necessary to test its inversion capability of recovering the preset signal model, especially in the existence of random noise. In this section, a toy problem, the artificial signal $x(t)=5+12 \sin (2 \pi t+(\pi / 4))+5 \cos (4 \cdot 2 \pi t)+r(t)$ (shown in Fig. 4(a)) is taken to test the inversion capability, where $t \in[0,1.1]$, sampling interval $\Delta t=0.005$, and the uniform random vector $r(t) \in[-5,5]$ (to simulate the white noise).

First, the power spectral density of the artificial signal is calculated to determine the parameter $m$ (see Fig. 4(b)). It shows that there are two low frequency waves in this segment of the signal - one is about $0.9 \mathrm{~Hz}$, and the other is around $3.6 \mathrm{~Hz}$. The starting point of the power-spectral curve is not zero, which means that the DC component is not negligible. Therefore, the model of the signal is formulated as

$$
x(t)=f+A_{1} \sin \left(2 \pi \omega_{1} t+\varphi_{1}\right)+A_{2} \sin \left(2 \pi \omega_{2} t+\varphi_{2}\right)+\varepsilon
$$

Next, the coefficient optimization process using LDSE to determine the coefficients is carried out. According to the range of the artificial signal, the searching range is confined to $f \in[-15,15], A_{i} \in[0,15], \omega_{i} \in[0,15], \varphi_{i} \in[0, \pi]$, where $i=1,2$. The control parameters of LDSE are set as follows. The population size $N=30$, and the adsorption probability [6] $p_{a}=0.5$, tolerate error $\epsilon=0.001$. A typical convergence history of LDSE is presented in Fig. 5.

To evaluate the influence of white noise, 100 runs are executed independently with different random seeds for generating $r(t)$. The global optimization algorithm LDSE can converge for all cases. The statistical results of its convergence history are given as follows. The iteration used for each run ranges from 67 to 836, and the number of function evaluations (nfe) ranges from 3259 to 41876. The average of nfe is 5287.2, and the standard deviation of nfe is 2694.8. Accordingly, we get the confidence level of their coefficients as follows. $f=4.99 \pm 0.067, A_{1}=12.00 \pm 0.026, \omega_{1}=1.00 \pm 0.015$, $\varphi_{1}=0.785 \pm 0.0033, A_{2}=5.01 \pm 0.028, \omega_{2}=4.01 \pm 0.022, \varphi_{2}=1.57 \pm 0.031$ with confidence level $95 \%$. Since all the coefficients have been determined, low frequency waves could be separated and removed from the original signal. The separated results are shown in Fig. 6. Two sine waves are marked as blue crosses and triangles respectively. The remained signal is marked as a red polygonal line, which contains the DC component $f(=5.0$ in this case) and the white noise $r(t)$. 
a

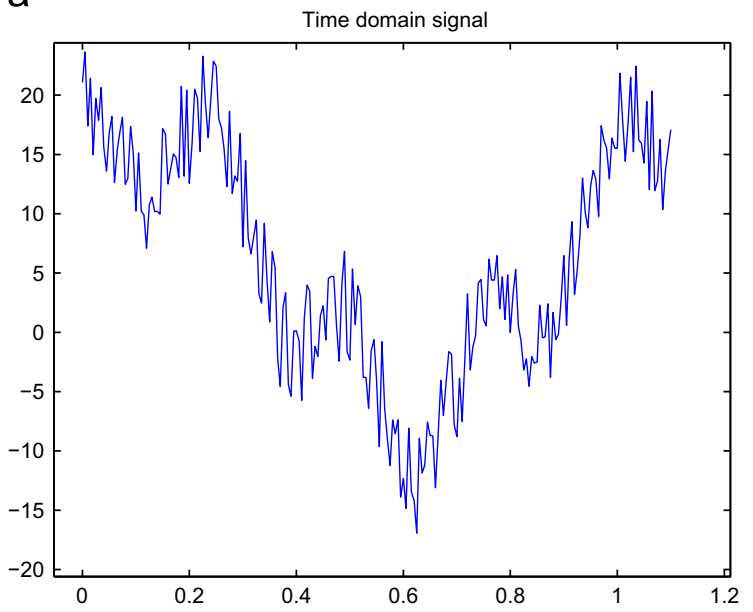

b

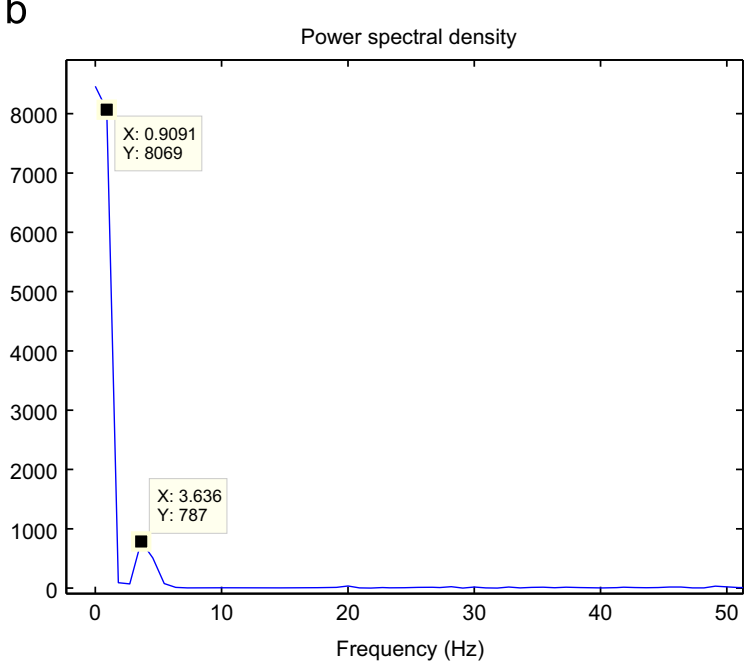

Fig. 4. An artificial signal and its power spectral density. (a) Time domain signal (b) Power spectral density.

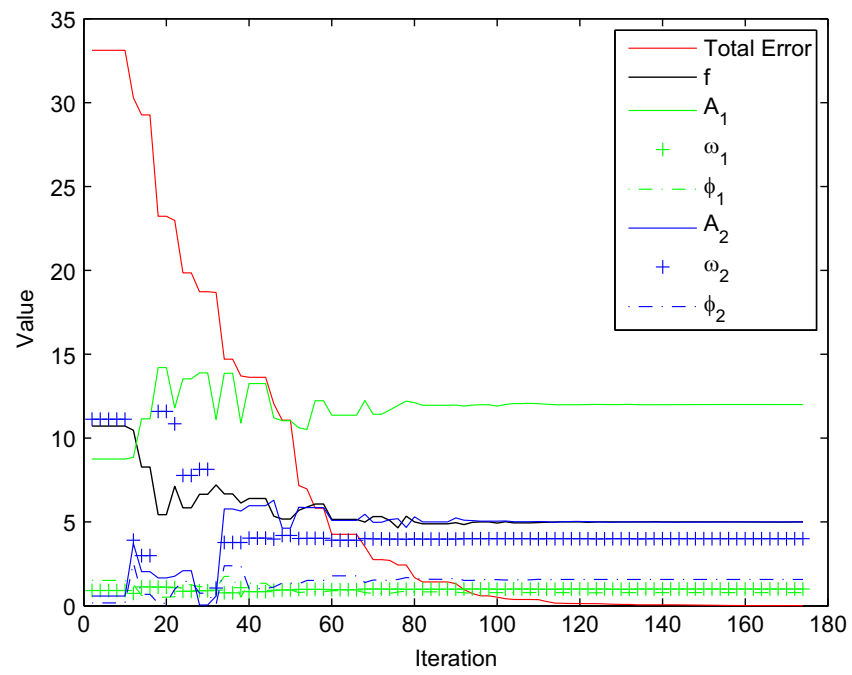

Fig. 5. A typical convergence history of LDSE.

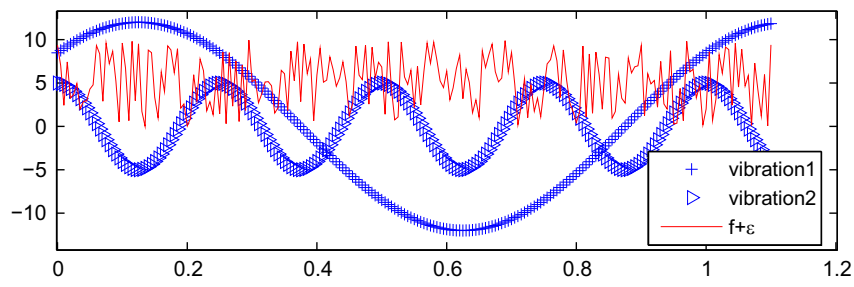

Fig. 6. A typical decomposition result of the artificial signal.

As an ideal case of the above signal, when there is no white noise, i.e., $r(t) \equiv 0$, a similar graph of power spectral density in Fig. 4(b) could be obtained, so the parameter $m=2$ as well. By LDSE algorithm, we get

$$
f=5.0000, \quad A_{1}=12.0000, \quad \omega_{1}=1.0000, \quad \varphi_{1}=0.7854, \quad A_{2}=5.0000, \quad \omega_{2}=4.0000, \quad \varphi_{2}=1.5708 .
$$

The coefficients are almost exactly the same as their preset values in the model.

These results show that the proposed wave system fitting can reconstruct the preset model reliably, and the method is stable with the support of global optimization. 


\section{Discussion}

So far we have shown how to separate and remove the signals of low frequency vibrations by wave system fitting (WSF). The readers may wonder why not just use the low-pass filter instead of WSF, why damping is not considered in the model of balance signal, why not use the frequencies directly from finite element analysis, or fft of knocking test signal, whether it is necessary to choose complete cycles of signal for WSF analysis, etc. Such questions had been our confusion too. The answers are as follows.

(a) Low-pass filter cannot get the correct DC component $f$ when the sampling signal is not a complete cycle of vibration. As a result, the resulting value of aerodynamic force could be wrong. For example, for the artificial signal in Section 3 without white noise (i.e., $r(t) \equiv 0$ ), the low-pass filter [10] will give $f=6.1056$ (the average value of the filtered signal), but the correct $f$ is 5.0. In fact, this misleading result is inevitable whenever the sampling signal is not a complete cycle of vibration. The error might be reduced if the signal has more vibration periods, and the sample signal is carefully truncated to approximate complete cycles. But it is very difficult, if not impossible, in practice. First, the test time duration in shock tunnels is not long enough to get more vibration periods. It is usual that the vibration with lowest frequency can only last 1-4 periods before the steady test is over. Second, the vibration of the model-balance-sting system has more than one frequency $\omega_{i}$, and the waves have different initial phases $\varphi_{i}$. The composite wave shape could be very complex. In addition, random noise could increase the complexity of signal wave shape. This makes it more difficult to choose which part of the balance signal should be truncated for analysis.

(b) The frequency information of knocking test or FEA is an important reference for modeling the balance signal. However, they can not directly replace $\omega_{i}$ in Formula (1) because there are many frequency vibrations, but only a few of them could be excited in one wind-tunnel shot, and it is difficult to tell which ones have been excited, though the balance signal is out there.

(c) The fft (or power spectral density) of wind-tunnel test signal can provide good initial values of $\omega_{i}$ in the model of the balance signal, but replacing them should be careful. As can be seen from Fig. 4, the lower frequency should be 1.0, but the power spectral density gives 0.9091 , which will cause much error to the result. Do so only if it is necessary, i.e., no other better choice. For example, sometimes, the balance signal has less one complete cycle during the nominal $100 \mathrm{~ms}$ because the test model is too heavy, or the model-balance-sting system is not stiff enough. In this case, the next 100-150 ms could also be used to identify the lowest frequency of balance signal, because the mode and shape of vibration will not change much in the next 100-150 ms, though the test flow is not steady anymore.

(d) Damping is negligible during the nominal $100 \mathrm{~ms}$ for shock-tunnel tests. In our earlier study, we have considered a model with damping terms, $x(t)=f+\sum_{i=1}^{m} A_{i} e^{-k_{i} t} \sin \left(2 \pi \omega_{i} t+\varphi_{i}\right)+\varepsilon$. But the results show that it performances no better than Formula (1) in all cases. In fact, the damping in shock tunnels is relatively very small comparing to the stiffness of the force measurement system, and the test time is rather short. Therefore, the more concise model, Formula (1), is preferred.

(e) Choosing complete cycles of the signal is no longer a necessary process in WSF. Here, by "the cycle of the signal is complete" we mean the number of vibration periods in the sampling window is an integer. For the above mentioned artificial signal (see Figs. 4 and 6), the cycles of the two low frequency vibration are 1.1 and 4.4 respectively, which are both considered to be incomplete. If $10 \%$ of the signal is cut off from the beginning or the end, the remained signal is considered having complete cycles of vibration. In practical application, cycle averaging is an easy to understand and easy to use method, in which the balance signal is truncated into complete cycles and then averaged directly to approximate the DC component $f$. In theory, the result should be the same as that of WSF if there is no random noise and the cycle of the chosen signal is complete. However, this is impossible in practice. It is hard to see the starting and/or ending point of a vibration cycle due to the complexity of the vibration mode and random noise, as mentioned in Discussion (a). The cycle choosing is a dilemma, and the subjectivity is inevitable when choosing by hand. In WSF, the cycle choosing is not necessary any more. In theory, if there is no random noise, the signal model can be reconstructed if only we have a segment of its sampling value. This is perhaps the most significant feature of WSF. However, the random noise is always inevitable in practice. It could distort the desired DC component $f$ if the sampling signal is too short to describe the vibration mode. In our practical applications, we find the sampling signal should contain at least more than a half cycle of vibrations for WSF to get a reliable result, even though the lowest vibration frequency is known. The degree of this requirement depends on the noise level of each wind tunnel. Therefore, the users are encouraged to use the balance signal as more as possible in WSF, if only the test flow around the model-balance-sting system is steady, to reduce the negative effect of the noise.

\section{Application in shock tunnel JF-12}

Before the wave system fitting (WSF) is designed, cycle averaging is used for the force measurements. The results are good enough for test models of small scale, because the frequencies of the vibrations are all high, and many cycles of the signal could be sampled. Cycle averaging had been worked fine until some interesting signals turned up. A typical example is 
the pitch moment signal of shot \#20121225 (see the left-top of Fig. 7). The signal seemed odd to us. The strange signal motivated us to study the relationship between balance signal and the vibration mode of the model-balance-sting system. Thereafter the signal decomposition method, WSF, was designed. The decomposition result show that two vibrations with
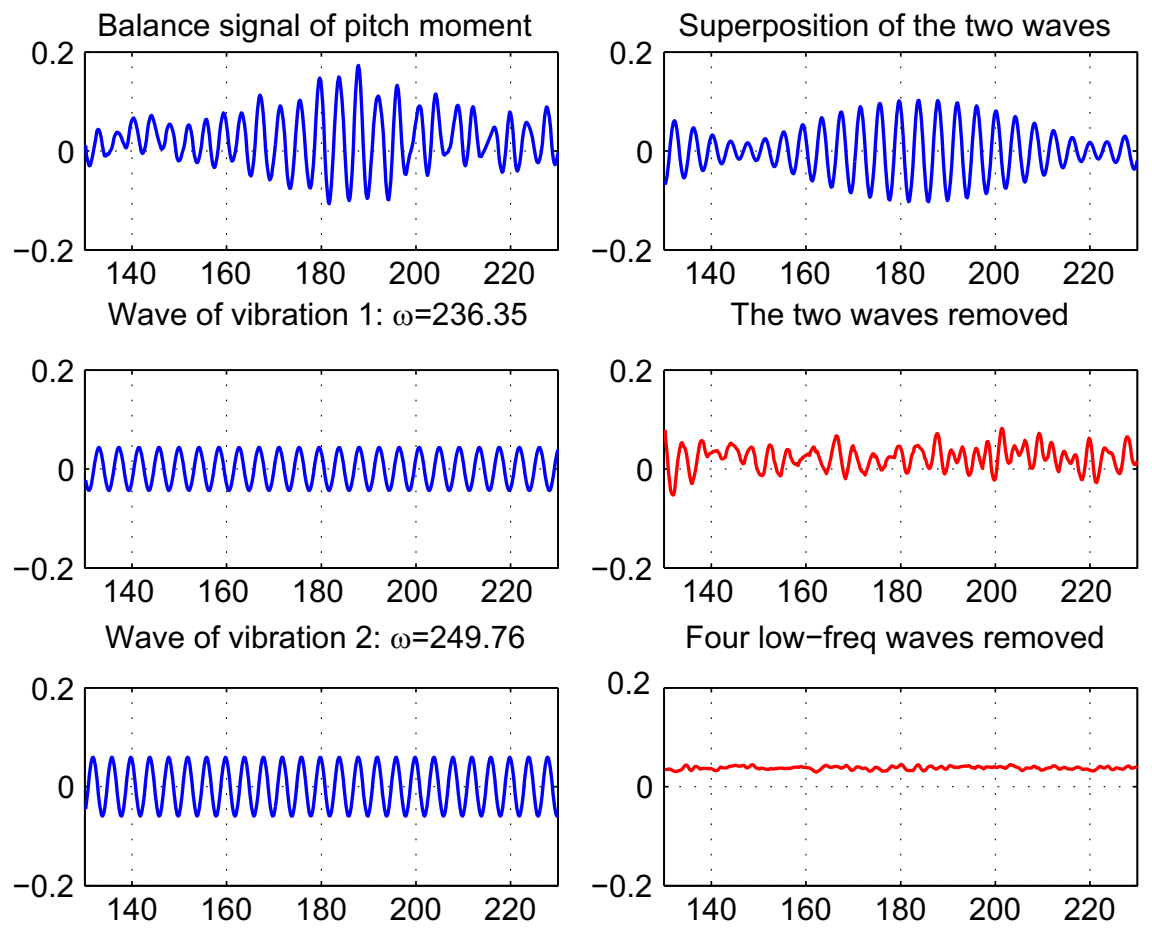

Fig. 7. Decomposition of a pitch moment signal, time interval: $130-230 \mathrm{~ms}$.

a
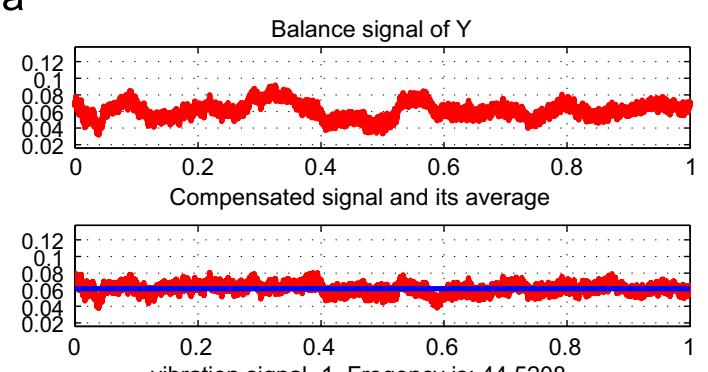

vibration signal-1, Freqency is: 44.5208

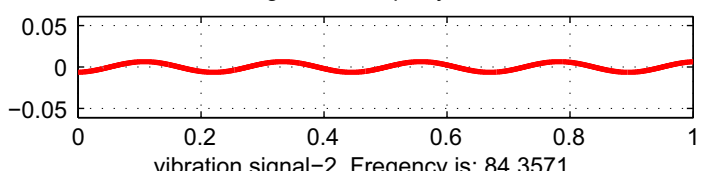

vibration signal-2, Freqency is: 84.3571

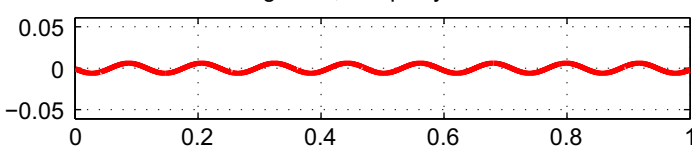

vibration signal-3, Freqency is: 30.808

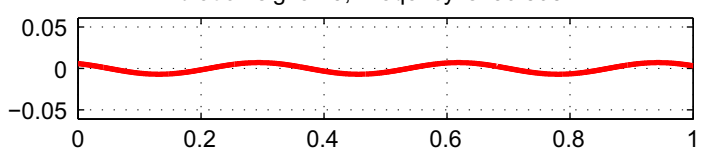

b
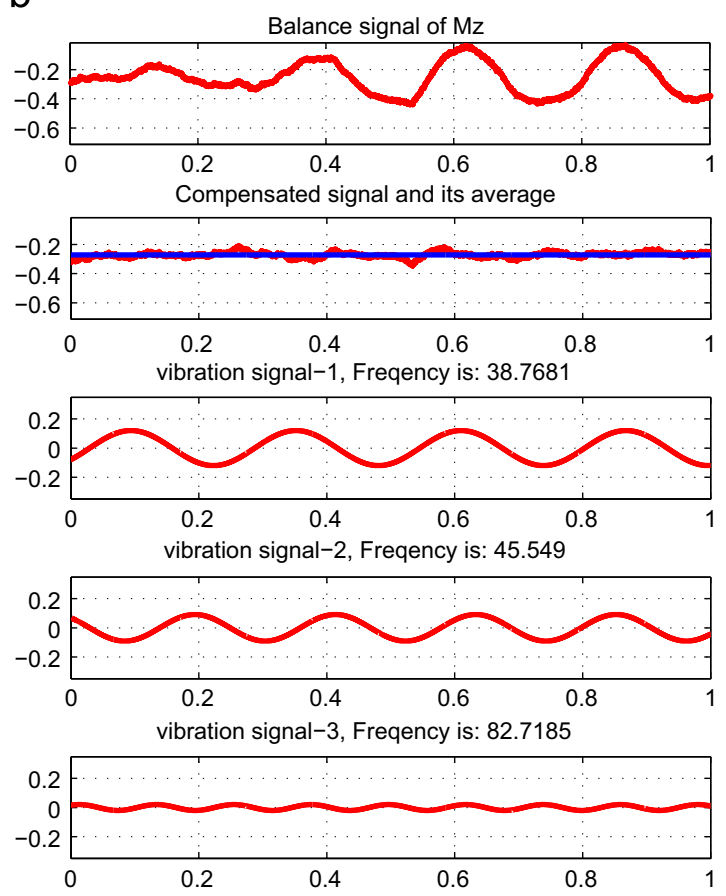

Fig. 8. Typical decomposition results of balance signals, time interval: 140-240 ms. (a) Decomposition of normal force signal. (b) Decomposition of pitch moment signal. 


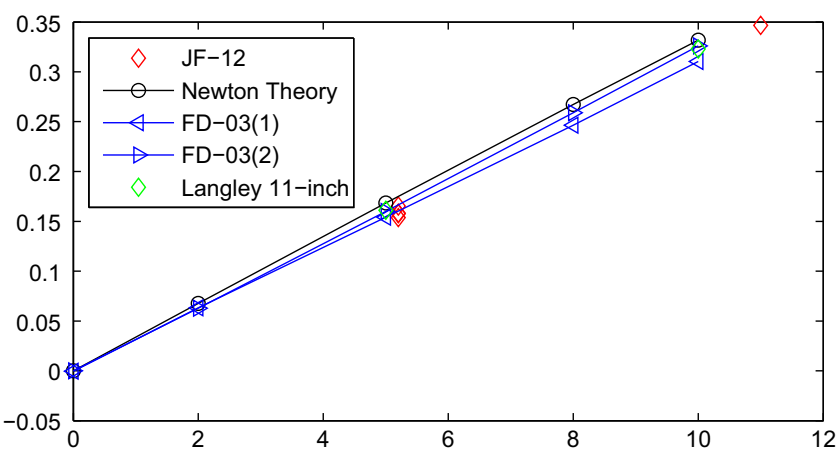

Fig. 9. Coefficients of normal force of sharp cone at different angles of attack.

slightly different frequencies, $236.35 \mathrm{~Hz}$ and $249.76 \mathrm{~Hz}$, were exited and the superposition of the two vibration waves looks like resonance phenomenon (see the right-top of Fig. 7). After the two waves are removed, the signal becomes familiar as usual. If necessary, more steady signal could be obtained by removing more vibration waves (see the right-button of Fig. 7).

However, as the model scale became larger, cycle averaging encountered the cycle choosing problem mentioned in the Discussion (e), and the WSF became necessary to get high accuracy results. Now the WSF has been coded as a standard tool for post-processing of balance signals. Typical decompositions of balance signals with the WSF-tool is illustrated in Fig. 8 . The balance signal in axis direction is not shown here because its vibration has much higher frequencies than that in the normal direction, and the measurement accuracy is already acceptable by cycle averaging, though WSF could further improve the accuracy.

For the large cone model mentioned in Section 2.1, several wind-tunnel tests have been done in JF-12, and the comparison result of its normal force coefficients at different angles of attack with Newton's theory and other wind-tunnels [4] is shown in Fig. 9, in which the nominal Mach numbers of all shots are the same, i.e., 7, but the actual Mach numbers are inevitably slightly different. It shows that the force measurement result by WSF is reliable, though the lines/points of different shock tunnels do not coincide with each other due to differences of other parameters such as Reynolds number and the actual Mach number, which are beyond the scope of this paper.

Besides the sharp cone, a number of other test models have also experimented in JF-12 to measure their aerodynamic force coefficients, and all of their balance signals were decomposed with WSF. But the results are for engineering purpose only, and omitted here.

\section{Conclusion}

The test duration of JF-12 is long enough for high speed data acquisition system to get periodic signals, but still needs special techniques to get force-measurement results with high accuracy. Meanwhile, some interesting but puzzling signals (such as the signal in Fig. 7) need explanations. To this end, a new signal decomposition method, wave system fitting (WSF), has been presented. It can separate and remove vibration waves of low frequency without influence on the DC component, and it does work no matter the cycle of the sample signal is complete or not. It is specially useful and irreplaceable whenever only a few cycles of vibration signal could be obtained, as is usual for shock tunnels. Of course, the proposed force-measurement method WSF is suitable not only for JF-12, but also for any other shock tunnels, especially for those with long test duration. WSF is now a standard post-processing tool for JF-12, and the results show that it works reliably with high accuracy.

Only a part of the balance signal, corresponding to the steady test phase (nominal $100 \mathrm{ms),} \mathrm{is} \mathrm{used} \mathrm{in} \mathrm{WSF.} \mathrm{The} \mathrm{signal}$ segment during the starting up of the wind tunnel and flow establishing process (20-30 ms in JF-12) is ignored so far. It is obviously a waste of valuable information. Analysis on this part of the signal is a meaningful but challenging topic left for our future studies.

\section{Acknowledgements}

This research has been supported by Innovation Grant of Chinese Academy of Sciences and the National Natural Science Foundation of China (Grant No. 90916028).

\section{References}

[1] A.X. Collopy, S.W. Lee, E.C. Marineau, Development of Dynamic Force Measurement Capabilities at AEDC Tunnel 9, AIAA Paper, $2014-0983$.

[2] Z. Jiang, H. Yu, Experiments and development of Long-test-duration Hypervelocity Detonation-driven Shock Tunnel (LHDst), AIAA Paper, 2014-1012.

[3] D.D. Joshi, P. Vadassery, F.K. Lu, Acceleration Compensation for Drag Measurements in Hypersonic Shock Tunnel, AIAA Paper, $2013-1020$. 
[4] C.L. Ladson, Air-Helium Simulation of the Aerodynamic Force Coefficients of Cones At Hypersonic Speeds, NASA Technical Note D-1473, 1962.

[5] C. Luo, B. Yu, Low dimensional simplex evolution: a new heuristic for global optimization, J. Glob. Optim. 52 (1) (2012) 45-55.

[6] C. Luo, S.-L. Zhang, B. Yu, Some modifications of low dimensional simplex evolution and their convergence, Optim. Methods Softw. 28 (1) (2013) 54-81.

[7] C. Luo, C. Wang, Z. Jiang, S.-L. Zhang, A metamodel-assisted evolutionary algorithm for expensive optimization, J. Comput. Appl. Math. 236 (2011) 759-764.

[8] E. Marineau, M. MacLean, E. Mundy, M. Holden, Force measurements in hypervelocity flows with an acceleration compensated strain gage balance, J. Spacecr. Rock. 49 (3) (2012) 474-482.

[9] K.W. Naumann, H. Ende, G. Mathieu, Technique for aerodynamic force measurement within milliseconds in shock tunnel, Shock Waves 1 (3) (1991) 223-232.

[10] T. O'Haver, 2014. iFilter: Interactive Fourier Filter, version 4.0, Available at: 〈http://terpconnect.umd.edu/ toh/spectrum/InteractiveFourierFilter.htm〉.

[11] M.J. Robinson, D.J. Mee, C.Y. Tsai, R.J. Bakos, Three-component force measurements on a large scramjet in a shock tunnel, J. Spacecr. Rock. 41 (3) (2004) 416-425.

[12] N. Sahoo, K.P.J. Reddy, Force measurement techniques for hypersonic flows in shock tunnels, Int. J. Hypersonics 1 (1) (2010) 31-58.

[13] W. Silver, C.V. Stahle, C.G. Stouffer, Inertia-compensated balance for wind-tunnel buffet measurements, J. Spacecr. Rock. 1 (6) (1964) 690-691.

[14] M. Takahashi, K. Komuro, K.H.T., Itoh, S. Ueda, Development of a New Force Measurement Method for Scramjet Testing in a High Enthalpy Shock tunnel, AIAA Paper, 1999-4961, 1999. 